\title{
Long-Term Maize-Legume Intercropping Shifts Structure and Composition of Soil Microbiome with Potential Impacts on Ecosystem Services and Food Safety
}

Aneth Mwakilili ( $\nabla$ anethdavid367@gmail.com )

University of Dar es Salaam https://orcid.org/0000-0002-1633-297X

Kilaza Samson Mwaikono

Dar es Salaam Institute of Technology (DIT)

Sebastian Larsson Herrera

Swedish University of Agricultural Sciences (SLU),

Charles Midega

International Centre for Insect Physiology and Ecology (ICIPE)

Francis Magingo

University of Dar-es-Salaam (UDSM)

Beatrix Alsanius

Swedish University of Agricultural Sciences (SLU)

Teun Dekker

Swedish University of Agricultural Sciences (SLU)

Sylvester Leonard Lyantagaye

University of Dar es Salaam Mbeya College of Health and Allied Sciences (UDSM-MCHAS)

\section{Research Article}

Keywords: Soil microbiome, push-pull farming, intercropping microbiome, 16S, ITS, Desmodium spp. intercrops

Posted Date: February 12th, 2021

DOl: https://doi.org/10.21203/rs.3.rs-221776/v1

License: (9) (1) This work is licensed under a Creative Commons Attribution 4.0 International License. Read Full License 
Version of Record: A version of this preprint was published at Plant and Soil on August 14th, 2021. See the published version at https://doi.org/10.1007/s11104-021-05082-w. 


\section{Abstract}

Purpose: Push-pull is an intercropping technology that rapidly spreads among Sub-Saharan smallholder farmers. It intercrops maize with Desmodium to fight off stem borers, eliminate parasitic weeds, and improve soil fertility and yields. The above-ground components of push-pull cropping have been well investigated. However, impact on and from the soil microbiome and its role in diverse ecosystem benefits are unknown. Here we describe the soil microbiome associated with push-pull and compare it with maize monoculture.

Methods: Soil samples from long-term maize-desmodium intercropping and maize monoculture plots were analysed using $16 \mathrm{~S}$ and ITS metagenomics.

Results: Maize-desmodium intercropping caused a strong divergence in fungal microbiome, which was more diverse and species rich than monoculture plots. Zooming into genera revealed that intercropping enhanced fungal genera linked to important ecosystem services. These include mycorrhizal and endophytic groups such as Edenia, Acrocalyma and Colletotrichum, saprophytic and decomposer fungi like Pithya and Cristinia and fungi with biocontrol properties, for instance, Talaromyces, Penicillin, Clonostachys and Trichoderma. Fungal genera enriched in monoculture plots were few, and were functionally linked to plant diseases (for example Didymella, Curvularia and Parastagonospora), and human pathogenic taxa (Exserohilum, Curvularia and Aspergillus). Although separating well, bacterial microbiomes did not, except in a few genera, differ between treatments.

Conclusion: Maize-desmodium intercropping diversifies fungal microbiomes and favors taxa that are associated with important ecosystem services, including plant health, productivity and food safety. Further studies should increase the resolution of shifts noted above, experimentally ascertain the inferred functions, and translate this knowledge to improve cropping systems.

\section{Introduction}

In this study, push-pull technology was used as a model for understanding interactions between plants and soil microbes in intercropping farming systems, and the potential impacts of the soil microbiome on plant health and productivity. Push-pull technology is an ecological habitat management strategy for the control of major pests of cereals, particularly maize and sorghum. Since its inception in the 90's, the technology has spread to smallholder farmers across southern and eastern Africa who use the technology to manage stem borers and fall armyworms attack on cereal crops thus increasing yield (Midega et al. 2018). The technology exploits the chemical ecology of leguminous intercrops belonging to the genus Desmodium and grass trap crops such as Napier or Brachiaria spp. The intercrop "pushes" stem-boring insects from the main crop through release of volatile compounds that signal an unfavourable egg-laying environment. At the same time, the trap crop "pulls" the insects towards itself but does not support their development (Khan et al. 2003; Khan et al. 2010). Both Desmodium and trap crops are a reliable source of animal fodder particularly in drought periods as farmers do not uproot them 
between farming seasons (Khan et al. 2010). The technology is effective and importantly, affordable for smallholder farmers in Sub-Saharan Africa.

However, over the years several additional benefits of push-pull cropping were uncovered. These indicate diverse soil-based mechanisms and warrant further study. It was found that Desmodium employs allelopathic mechanisms that dramatically reduce infestation of the parasitic weed Striga hermonthica to cereal crops, further adding to yield increases (Khan et al. 2002). Moreover, push-pull technology provides other ecological benefits contributing to improved cereal crops yield, including nitrogen fixation by Desmodium, soil structure improvement and mulching effect in the fields. Finally, push-pull cropping reportedly reduced the incidence of human pathogenic fungal toxins in maize kernels (Njeru et al. 2020; Owuor et al. 2018).

Whereas many components of the push-pull farming technology and their underlying mechanisms have been investigated, one of the areas that has received no attention is the impact of push-pull cropping systems on the soil microbiome and vice-versa. Soil microorganisms are known to promote plant health and productivity through direct and indirect mechanisms mediated through root systems (van der Heijden et al. 2008). Therefore, it is of interest to explore the contribution of soil microorganisms on the effectiveness of the push-pull system.

A better understanding of soil microbial diversity in intercropping agricultural practices is necessary for optimal exploitation of their potential ecosystem benefits in plant health and productivity. Eventually, beneficial plant growth and protection activities of soil microorganisms may be incorporated into sustainable food production, offering perspectives on reducing the reliance on and environmental impacts of agrochemical inputs. The first step towards this understanding is to characterize the microbial diversity and how this has evolved through changing from monoculture to polyculture cropping.

Microbiome studies are increasingly used to discern potential impacts of farming practices such as intercropping on structure and diversity of soil microbiota, in addition to other benefits, such as increase in yield and mineral nutrients availability. A study by Debenport et al. (2015) found an increase in diversity of soil bacterial and fungal communities around the millet rhizosphere when intercropped with woody shrubs compared to monocultures. In another study, an increase in yield as well as overall diversity of soil bacteria was also observed in maize-peanut intercropping systems. In addition, the abundance of beneficial soil bacteria was higher in intercropping systems with complete and semi-below ground interactions compared to monoculture systems ( $\mathrm{Li}$ et al. 2018). Tang et al. (2020) observed variations in availability of soil minerals and enzymes as a result of intercropping with either peanut or cassava. In the same study, certain bacterial groups including Nitrospirae increased in abundance, and others were associated with improved soil quality.

The current study compared the broad diversity of soil microorganisms between long-term maizedesmodium intercropping (push-pull system without the trap crop) and maize monoculture plots. Specifically, 16S and ITS amplicon sequencing were used to investigate the differences in the soil bacterial and fungal population structures between long-term maize-desmodium intercropping and maize 
monoculture practices in a context of potential ecological benefits. The mapping of the microbiomes of these maize monoculture and intercropping plots demonstrated that particularly the fungal microbiome was highly diversified in maize-desmodium intercropping plots compared to maize monoculture plots. The results are discussed in the context of reported 'benefits' around maize-desmodium intercropping systems, and known ecological functions of taxa contributing to the observed difference have important ecological functions. Further studies are recommended to discern key determinants of the observed differences, their importance in ecosystem (dis)services. Knowledge and translation of this knowledge into other cropping systems could advance sustainable food production through fostering belowground microbial communities that support plant health and productivity.

\section{Methodology \\ Sampling site}

To compare soil microbial profiles between maize-desmodium intercropping and monoculture maize farming, we obtained soil samples from long-term experimental plots at the International Centre for Insect Physiology and Ecology (ICIPE), Mbita campus, Kenya ( $\left(0^{\circ} 25.877 \mathrm{~S} 34^{\circ} 12.425 \mathrm{E}\right)$.

\section{Soil samples}

Soil samples were collected from three long-term experimental plots that have been continuously maintained as pairs of maize-desmodium intercropping and maize monoculture each since 1998, 1999 or 2003. We collected three samples from maize-desmodium intercropping plots established each year and four samples from each monoculture plot.

For each individual sample, sampling was done by randomly taking three 15-18 cm deep cores across the plot between rows of maize as close to Desmodium spp. root system as possible. Afterwards, each soil sample was homogenized and sieved through a $4 \mathrm{~mm}$ wire mesh. About $200 \mathrm{~g}$ soil sub-sample was collected and stored at $-20^{\circ} \mathrm{C}$ until further processing.

\section{DNA extraction and sequencing}

DNeasy Powersoil kit (Qiagen, Manchester, UK) was used for total DNA extraction from the soil samples following the manufacturer's protocol. Briefly, $0.25 \mathrm{~g}$ soil was added to PowerBead Tubes containing a lysis buffer and vortexed for a few seconds. The resulting mixture was centrifuged at $10,000 \mathrm{~g}$ for $30 \mathrm{~s}$ before discarding the pellet and centrifugation of the supernatant in spin columns. Tris- $\mathrm{HCl}$ solution was used to wash off DNA from the spin column. Nanodrop spectrophotometer and gel electrophoresis were used to assess the quality of the extracted DNA. The DNA samples were stored at $-20^{\circ} \mathrm{C}$ until further processing.

DNA sequencing was done at Inqaba Biotechnical Industries (Pty) Ltd (Pretoria, South Africa). The primers targeting V1-V3 of the 16S rDNA gene of the bacteria (27F and 518R primer pairs) were used to amplify DNA under the following PCR conditions: initial denaturation at $95^{\circ} \mathrm{C}$ for 2 minutes, followed by 
30 cycles of denaturation at $95^{\circ} \mathrm{C}$ for 30 seconds, primer annealing at $60^{\circ} \mathrm{C}$ for 30 seconds, and extension at $72{ }^{\circ} \mathrm{C}$ for 30 seconds, with a final elongation at $72{ }^{\circ} \mathrm{C}$ for 5 minutes. For fungi, ITS1F and ITS2 primer pairs targeting the ITS1 region was used for PCR amplification under the following conditions: $95^{\circ} \mathrm{C}$ for 2 minutes, followed by 30 cycles of denaturation at $95^{\circ} \mathrm{C}$ for 30 seconds, primer annealing at $50{ }^{\circ} \mathrm{C}$ for 30 seconds, and extension at $72{ }^{\circ} \mathrm{C}$ for 1 minute. Final elongation was held at $72{ }^{\circ} \mathrm{C}$ for 5 minutes.

Resulting amplicons were gel purified, end repaired and illumina specific adapter sequence were ligated to each amplicon (NEBNext Ultra II DNA library prep kit). Following quantification, the samples were individually indexed (NEBNext Multiplex Oligos for illumina Dual Index Primers Set 1), and another AMPure XP bead based purification step was performed. Amplicons were then sequenced on Illumina's MiSeq platform, using a MiSeq v3 kit with 600 cycles (300 cycles for each paired read and 12 cycles for the barcode sequence) according to the manufacturer's instructions. Demultiplexed 300 bp paired-end reads were obtained.

\section{Bioinformatics and statistical analysis}

FASTQC (Wingett and Andrews 2018) was used to assess the quality of raw sequence reads. Then, QIIME2 v2020.8 suit of packages was used for quality control, construction of a feature table, taxonomic classification and diversity analyses (Bolyen et al. 2019). Briefly, the dada2 plugin (Callahan et al. 2016) was used to trim and truncate poor regions of both the $16 \mathrm{~S}$ and ITS raw reads. The truncation and trimming was set to -p-trim-left-f 8, --p-trim-left-r 8; and -p-trunc-len -f 290, -p-trunc-len-r 260, for the 16S; while for the ITS, parameters used were p-trim-left 22, -p-trunc-len 299. Bacterial taxonomic assignment was done using feature-classifier classify-sklearn (Bokulich et al. 2018; Pedregosa et al. 2011), only including reference genes that was classified to at least genus level, by using SILVA v.138 99\% database (Quast et al. 2013) pre-trained to V1-V3 region of the 16S. For the ITS, we used UNITE v8.2 reference database (Nilsson et al. 2019) pre-trained to ITS1.

Further, the feature table was converted into biom format (using qiime 2 export tool), and then imported into R (R Core Team 2020) using "qiime2R" (Bisanz 2018). For visualising the number of ASV, genera, families and orders present in the dataset we filtered out everything that was present only once at each level and then Venn diagrams were produced using function vennCounts form package "limma" (Ritchie et al. 2015). Then, everything that was unassigned at family level was filtered out. Genera that were present in either all samples, in one treatment from one area or were present in more than $60 \%$ in the samples from either maize or maize-desmodium intercropping were kept.

For constructing dendrograms, primary component analysis (PCA) and heatmaps data was transformed using CSS (cumulative sum scaling) by using a package "metagenomeseq" (Paulson et al. 2013). To perform a principal component analysis (PCA), we used package "recipes" (Kuhn and Wickham 2020), and annotated ellipses using a Khachiyan algorithm from package "ggforce" (Pedersen 2020).

Dendrograms were constructed using a jaccard index from package "vegan" (Oksanen et al. 2020) with a presence absence standardization and plotted using "ggtree" (Guangchuang et al. 2017). 
Species diversity (Shannon) and richness (chao1) were calculated on untransformed and unfiltered data using "vegan" through the package "phyloseq" (McMurdie and Holmes 2013), while evenness was calculated as the Shannon index divided by the natural logarithm of the total number of species. All indices were tested for significance using a Student's t-test.

Differential expression analysis was done on untransformed but filtered data based on a negative binomial distribution through "DESeq2" (Love et al. 2014). The resulting log2fold changes were shrunken using the adaptive shrinkage estimator from package "ashr" (Stephens et al. 2020). Genera were deemed to significantly impact treatments if they had an adjusted p-value smaller than 0.05 (Wald test), and an absolute log2fold change of over one, which was then visualised on a volcano plot modified from package "EnhancedVolcano" (Blighe et al. 2020). The result from the differential expression analysis also was used to group data in the heatmap and label significant genera in the PCA. All data was manipulated using "tidyverse" (Wickham et al. 2019) and visualised using "ggplot2" (Wickham 2016).

\section{Results}

Below we describe some of the major differences found between plots that have been under long-term maize-desmodium intercropping or monoculture management. The results outline the differences in composition and abundance of bacterial and fungal taxa, measures the diversity indices within and between plots and treatments, assesses the degree of separation, and highlights differences in taxa with known significance in plant and human health. In addition to the data presented here, more detailed information is deposited online.

\section{Composition and abundance of soil microbiome in maize- desmodium intercropping and monoculture plots}

A total of 1085 fungal ASVs distributed across 225 fungal genera were identified from mono-culture plots, whereas 1262 ASVs from 284 genera were identified in maize-desmodium intercropping plots. For bacteria, a total of 1934 ASVs were identified from monoculture plots and 1333 ASVs from maizedesmodium intercropping plots, distributed across 195 and 162 genera, respectively. Figure 1 shows the Venn diagrams of unique and common taxonomic units in maize-desmodium intercropping and monoculture plots. ASVs and genera that were present only in single samples were excluded from the Venn diagram. Maize-desmodium intercropping plots had a more diverse representation of fungal genera compared to maize monoculture plots (see also diversity indices, below).

In Fig. 2, the relative abundances of soil bacterial and fungal genera in both maize-desmodium intercropping and monoculture plots were plotted across the thirty most abundant genera. Not all bacteria were fully classified to genus level (Fig. 2A), whereas almost all fungi were classified to genus (Fig. 2B). Noteworthy is that several fungal genera, even though abundant to both cropping practices, significantly differed between the maize monoculture and push pull systems. These include Aspergillus (known human pathogens overrepresented in maize monoculture), Didymella (plant pathogens), Edenia (known 
beneficial genera overrepresented in maize-desmodium intercropping), Curvularia (plant pathogens), Albifimbria (saprophytic), Glomus (mycorrhizal), Parastagonospora (genus of many plant pathogens), and Achroiostachys.

The most abundant bacterial phyla were Acidobacteri, Actinobacteria, Chloroflexi, Proteobacteria, and Planctomycetes, whereas Ascomycota, Basidiomycota, Chytridiomycota, Glomeromycota and Mortierellomycota were the most abundant fungal phyla.

Diversity of soil microbiome between maize-desmodium intercropping and monoculture plots (alpha diversity)

No significant difference in diversity of soil bacteria genera was observed between monoculture and maize-desmodium intercropping plots $(p=0.246)$. In contrast, fungal genera were more diverse in maizedesmodium intercropping plots compared to monoculture $(p=0.047)$. Similarly, bacterial genera did not differ in richness $(p=0.238)$ and evenness $(p=0.206)$ between monoculture and maize-desmodium intercropping plots, whereas fungal general had significantly higher richness in maize-desmodium intercropping plots $(p=0.012)$. The evenness of fungal genera in maize-desmodium intercropping plots was not significantly different from that of monoculture plots $(p=0.386)$.

\section{Diversity of soil microbiome between maize-desmodium intercropping and monoculture plots (beta diversity)}

Figure 4 shows the impact of long-term maize-desmodium intercropping farming practice on composition of soil microbes at the genus level, which shows separation of bacterial and fungal profiles based on treatment (maize monoculture and maize-desmodium intercropping). Generally, bacterial communities did not separate the treatments strongly (Fig. 4A). In contrast, the effect of the cropping system on fungal microbial communities more strongly separated maize-desmodium intercropping and monoculture plots along the first principal component (Fig. 4B). It is notable that in addition to separation by treatment, plots from the same treatment separated out, particularly on PC2, albeit explaining roughly $10 \%$ of the variation.

\section{Differential abundance of soil microorganisms across maize-desmodium intercropping and monoculture plots}

Several fungal and bacterial genera showed varying abundances between maize-desmodium intercropping and monoculture plots and some occurred in one farming practice only. Figure 5 shows a heatmap plot of the abundance of bacterial (A) and fungal (B) genera both common and unique to each farming practice. The figure shows that fungal genera were relatively richly represented than fungal genera and were significantly higher in maize-desmodium intercropping plots than in maize monoculture plots (Fig. 5B), whereas there was a little differential expression levels of bacterial genera between the 
two farming practises (Fig. 5A). The plots separated largely by treatment (dendrogram, left hand panel of each heatmap plot).

More detailed analysis shows the differences in abundance at genus level (Fig. 6, supplementary tables 1 and 2). As can be inferred from the heatmap plot (Fig. 5A) and further reinforced here, fewer bacterial genera exhibited a differential abundance between maize-desmodium intercropping (push-pull) (1 genus) or monoculture plots (4 genera, Fig. $6 \mathrm{~A}$ ), whereas a larger number of fungal genera (52 genera) were significantly more abundant in maize-desmodium intercropping (push-pull) plots compared to monoculture plots (16 genera, Fig. 6B).

In maize-desmodium intercropping plots, fungal genera that were significantly more abundant represent a diversity of functional groups of which species have been described as saprophytic (most notably Pithya, Albifimbria, Acremonium, Pseudorobillarda and many less common taxa e.g. Cristinia), mycorrhizal and endophytic (most notably Edenia, Acrocalyma and Colletotrichum), and having biocontrol functions such as antifungal, antibacterial and/or nematicidal effects (most abundantly Edenia, Talaromyces, Penicillin, Clonostachys and Trichoderma). A few pathogenic genera were also enriched (most notably Mycoleptodiscus, a pathogen of legumes).

In contrast, maize monoculture plots were enriched in many genera with described plant pathogenic (Didymella, Curvularia, Parastagonospora, Tetracladium) or human pathogenic effects (Aspergillus, Curvularia, Exserohyllum), and only a few with known beneficial effects (most abundantly mycorrhizal genus Glomus, and endophytic Laetisaria).

\section{Discussion}

Intercropping is increasingly studied to provide sustainable alternatives to monoculture production systems. Intercropping provides diverse ecosystem services, some of which are immediate and pronounced (such as productivity, pollinator support, pest and disease reduction, nitrogen fixation (BybeeFinley and Ryan 2018; Nourbakhsh et al. 2019), whereas others are acquired over a longer time. Effects on and impact of the soil microbiome fall in the latter category and have been, in part for that reason, little studied. This particularly holds true for cereal crops, as they are frequently cropped annually and part of a rotational cropping scheme, which hampers long-term studies.

The maize-desmodium push-pull intercropping system, originally developed to reduce stem borer impacts (Khan et al. 2006), has evidently very pronounced below-ground benefits, including control of the parasitic weed striga, increase in soil carbon and fertility through perenniation and nitrogen fixation (Khan et al. 2016; Midega et al. 2018), and a lower incidence of mycotoxin-producing fungal infection (Owuor et al. 2018; Njeru et al. 2020). By virtue of the long-term cropping plots managed by ICIPE, long-term push-pull and monoculture plots (more than 14 years old) are exquisitely suited for studies on long-term effects of legume intercropping on soil microbiomes. 
In the current study, we demonstrate substantial qualitative and quantitative effects of long-term maize monoculture and maize-desmodium intercropping on structure and diversity of soil microbial communities. Below, we elaborate on the differences in bacterial and fungal microbiomes associated with these plots, and elaborate on differences of selected taxa with a known functional significance as plant symbionts or pathogens, or human pathogens.

\section{Diversity of cropping systems links to diversity in soil microbiome in maize-desmodium intercropping cropping}

Diversifying cropping systems, often using legumes as intercrop, may have been devised originally for purposes other than increasing biodiversity, such as food security, pest control (push-pull), green manure, or to avoid negative plant-soil feedback and soil legacy (Stagnari et al. 2017). However, ripple effects on biodiversity and other ecosystem services are apparent. Maize-desmodium push-pull intercropping is a good example of this. Initially designed to combat stemborers, it gradually revealed a range of additional ecosystem services, including combating parasitic weeds of cereals, increase soil nitrogen and carbon, and reducing incidence of mycotoxins in maize (Balaso et al. 2019; Cook et al. 2007; Xu et al. 2018). Here we add to these benefits a diversification of the soil microbial communities, with a particularly strong shift in the composition of fungal taxa, whose diversity was significantly higher in maize-desmodium intercropping than monoculture plots. By itself diversity in ecosystems is generally regarded as increasing stability, resilience and productivity (Prieto et al. 2015), mostly as a consequence of resource complementarity and functional redundancy (Cleland 2011; Rosenfeld 2002).

Whereas a perennial crop, such as Desmodium, could have shaped the rhizosphere and reduced biodiversity, intercropping may also lead to niche diversification in the rhizosphere and thereby increase biodiversity. Generally, research supports the later, i.e., an increased diversity of soil microorganisms in intercropping systems, particularly fungal microbes. For example, in wheat-soybean intercropping (Bargaz et al. 2017), maize/wheat-faba bean intercropping (Wang et al. 2020), millet-mung bean intercropping (Dang et al. 2020), and cucumber-cereal intercropping ( $\mathrm{Li}$ and Wu 2018). Tang et al. (2020), observed a higher diversity of both bacterial and fungal species in cassava-peanut intercropping, but also peanut monoculture, suggesting that the leguminous peanut intercrop was responsible for the observed higher soil microbial diversity, a factor we could not correct for in our study. Similarly, Gao et al. (2019) observed that peanut monoculture plots had the highest richness and abundance of soil bacteria compared to intercropping tobacco after two and four years. These observations, including the ones we made in the current study, indicate that the impact of legume intercropping on soil microbial communities is complex and system dependent. Regardless of the biodiversity, more critical is the functional biodiversity in terms of plant health and food safety, details of which we discuss below.

\section{Differential abundance of taxa and their potential functional significance}


Whereas in general many fungal taxa abundant in maize-desmodium intercropping plots appear to fulfill a saprophytic role (for example Arthrographis, Malbranchea, Lepiota, Cristinia and Pleurophragmium (supplementary Table II)), and thus may indicate higher carbon sequestration associated with the perenniation in these plots, other enriched fungal genera likely confer more distinct ecosystem services, such as Edenia, Talaromyces, Clonostachys, Trichoderma, Penicillium and Colletotrichum (supplementary Table II). Species belonging to these genera can form mycorrhizal and endophytic associations with plant roots that promote plant growth activities through increased nutrient supply, and/or directly or indirectly stimulate production of beneficial secondary metabolites and protection against pathogens and insect pests (Díaz-González et al. 2020; Hiruma et al. 2016; Macías-Rubalcava et al. 2008; Munasinghe et al. 2017; Ravnskov et al. 2006; Zin and Badaluddin 2020). Indeed, effects can be indirect and intricate: $T$. atroviride promoted growth and herbivory resistance of maize against Spodoptera frugiperda, possibly linked to induction of the jasmonic acid pathway leading to heightened induced defence (ContrerasCornejo et al. 2018). Similarly indirect, soil microbe composition and diversity was positively correlated to diversity and productivity of plants above ground in most systems (Schnitzer et al. 2011), which in turn would favor above ground biodiversity and biological control (Winqvist et al. 2011).

Although we do not know to what extent these direct and indirect interactions are functionally relevant in the push-pull systems, it is reasonable to assume that below ground intricacies do contribute to ecosystem services observed in push-pull and maize-desmodium intercropping systems, including above ground tritrophic interactions. For instance, in a recent study it was shown that maize grown on soil from push-pull plots showed a higher induced-defence response, including higher release of induced volatiles and lower herbivore damage (Mutyambai et al. 2019). Of particular notice in this context is the higher abundance of Edenia and Clonostachys species, which are associated with increased plant health, biocontrol and increased resistance against herbivore damage (lqbal et al. 2018; Macías-Rubalcava et al. 2008; Poveda et al. 2020).

Recent papers reported lower incidences of maize ear rot and associated mycotoxins (aflatoxins and fumonisins) (Owuor et al. 2018) as well as lower rate of infection of maize kernels with Fusarium verticillioides and Aspergillus flavus (Njeru et al. 2020) in smallholder farmers' push-pull plots compared to monoculture. Push-pull thus appears to promote food safety by reducing the risk of mycotoxins entering the human food chain, although the mechanisms remained unclear. We therefore were particularly keen on assessing the abundance of these fungal genera in soil samples of monoculture and maize-desmodium intercropping plots. Interestingly, a lower relative abundance of $A$. flavus was indeed associated with maize-desmodium intercropping cropping, whereas no association was found for $F$. verticillioides. The lower incidence of these ear rot infections thus may partially be explained by the abundance of the species in the soil. Additional factors, for instance related to the observed high fungal biodiversity, may further contribute to the reported lower mycotoxin incidence in push-pull plots. These may include fungal competition or biocontrol such as has been reported for Edenia, Myriococcum, Humicola, Penicillium, Clonostachys, and Trichoderma, which are enriched in maize-desmodium intercropping plots. Other studies have reported on the role of both bacteria (Venkatesh and Keller 2019) and fungal competition (Sarrocco et al. 2019; Wang et al. 2020) in reducing mycotoxins production. 
Further research on how mycotoxin incidence in maize kernels can be reduced by interactions between mycotoxin producing fungi and other soil microbes in maize-desmodium intercropping, would help in devising strategies to increase food safety through more healthy plant production systems.

Although the overall diversity of bacterial genera was not significantly different between the two farming practices, several genera were significantly abundant in maize-desmodium intercropping plots compared to monoculture. Most of them were unclassified, however, among those classified is a nitrogen-fixing soil bacteria genus Nitrospira spp (supplementary table I) (Koch et al. 2015). Enriched presence of Nitrospira spp. in maize-desmodium intercropping plots suggests involvement in nitrogen fixation, potentially through symbiosis with Desmodium spp, and thus an increased supply in Nitrogen that benefits maize plants in maize-desmodium intercropping plots.

\section{Concluding remarks}

This study evaluated the microbiome associated with maize-desmodium intercropping systems in order to understand its contribution to the well-described ecosystem services of these cropping systems. Understanding the complex interactions between plants, soil, microbiome and cropping systems is key in harnessing the benefits that are rendered to plant health and productivity. Whereas in some instances single genera and species appear to play key roles in protecting plants or promoting plant growth (Reva et al. 2019), many functions may arise through complex interactions within very diverse soil microbial communities, which give rise to emergent ecosystem properties that may not be readily assigned to functional roles of single taxa.

Intercropping systems, particularly those involving leguminous perennial components, promote complementarity in farming systems that facilitate effective land use in agriculture (Duchene et al. 2017). The various knock-on ecosystem effects of these polyculture systems have perhaps most clearly been described for maize-desmodium intercropping (Midega et al. 2010). Hitherto, the impacts on and contributions of the microbiome have, however, received little attention. We show significant interactions between maize-desmodium intercropping and the soil microbiome, with apparent ecosystem services in terms of diversity, production, plant health and food safety. Given the increasing availability of sequencing tools, we suggest that research on intercropping studies for improvement of crop health and productivity more integrally include metagenomics to assess the impact on and effects of the microbiome on plant productivity, plant health, and more broadly ecosystem health.

This study contributes to the extensive literature around push-pull technology through describing the bacterial and fungal microbiome associated with long-term monoculture and maize-desmodium intercropping cropping, and linking above ground benefits to below-ground microbial communities. It is a first step that should be further complemented with higher resolution analyses such as whole genome sequencing (WGS), to provide a finer-grained overview of functional groups of soil microorganisms. We further hope that future studies will experimentally dissect the contribution of the components of the microbiome identified here, in providing the diverse benefits reported (Midega et al. 2018), such that pushpull cropping techniques can be further improved, and translated to other cropping systems. 


\section{Declarations}

Funding: Funding for this research was provided by a Research Capacity Building Program from Swedish International Development Cooperation Agency (Sida) to UDSM (ADM, TD, SL) and the Swedish Research Council (621-2014-4816, TD).

Conflicts of interest/Competing interests: The authors have no conflicts of interest to declare that are relevant to the content of this article.

Consent to participate: NA

Consent for publication: NA

Availability of data and material: The datasets generated during and analysed during the current study are available in the Genbank Sequence Read Archive (SRA) repository under project number PRJNA667690 linked here https://www.ncbi.nlm.nih.gov/bioproject/PRJNA667690/.

\section{Authors' contributions}

Conceptualization and advice: TD, SL, ADM; Methodology and advice: TD, SL, ADM, BA, CM, FM; Formal analysis and investigation: TD, SL, ADM, KSM, SLH; Writing - original draft preparation: ADM, KSM, TD Writing - review and editing: all authors. Funding acquisition: TD, SL, FM Supervision: TD, SL, FM, CM, BA. All authors approved the submitted version for publication.

\section{Acknowledgements}

This research was performed with funding from Sida of Sweden through the University of Dar es Salaam, Tanzania, within the sub-programme 'Sustainable Agricultural Productivity - Processing and Value Chain to Enhance Food Security in Tanzania (ADM, TD, SL), and The Swedish Research Council (621-20144816, TD). We thank the laboratory and field staff at ICIPE Thomas Odhiambo and Duduville campuses for their generous support in sample collection and processing and DNA extraction, especially Dickens Nyagol, Levi and Maureen.

\section{References}

Balaso GM, Nalini C, Yankit P, Thakur P (2019) Push-pull strategy: novel approach of pest management. J Entomol \& Zool Stud 7(5): 220-223

Bargaz A, Noyce GL, Fulthorpe R, Carlsson G, Furze JR, Jensen ES, Dhiba D, Isaac ME (2017) Species interactions enhance root allocation, microbial diversity and $\mathrm{P}$ acquisition in intercropped wheat and 
soybean under P deficiency. Appl Soil Ecol 120:179-188

Bisanz JE (2018) qiime2R: Importing QIIME2 artifacts and associated data into R sessions. R package https://github.com/jbisanz/qiime2R. Accessed 01 Oct 2020

Blighe K, Rana S, Lewis M (2020) EnhancedVolcano: Publication-ready volcano plots with enhanced colouring and labeling. R package version $1.8 .0 \mathrm{https} / / /$ github.com/kevinblighe/EnhancedVolcano. Accessed 01 Oct 2020

Bokulich NA, Kaehler BD, Rideout JR, Dillon M, Bolyen E, Knight R, Huttley GA, Gregory Caporaso J (2018) Optimizing taxonomic classification of marker-gene amplicon sequences with QIIME 2's q2-featureclassifier plugin. Microbiome 6:90

Bolyen E, Rideout JR, Dillon MR, Bokulich NA, Abnet CC, Al-Ghalith GA, Alexander H, Alm EJ, Arumugam M, Asnicar F, Bai Y, Bisanz JE, Bittinger K, Brejnrod A, Brislawn CJ, Brown CT, Callahan BJ, CaraballoRodríguez AM, Chase J, Cope EK, Da Silva R, Diener C, Dorrestein PC, Douglas GM, Durall DM, Duvallet C, Edwardson CF, Ernst M, Estaki M, Fouquier J, Gauglitz JM, Gibbons SM, Gibson DL, Gonzalez A, Gorlick K, Guo J, Hillmann B, Holmes S, Holste H, Huttenhower C, Huttley GA, Janssen S, Jarmusch AK, Jiang L, Kaehler BD, Kang KB, Keefe CR, Keim P, Kelley ST, Knights D, Koester I, Kosciolek T, Kreps J, Langille MGI, Lee J, Ley R, Liu Y-X, Loftfield E, Lozupone C, Maher M, Marotz C, Martin BD, McDonald D, Mclver LJ, Melnik AV, Metcalf JL, Morgan SC, Morton JT, Naimey AT, Navas-Molina JA, Nothias LF, Orchanian SB, Pearson T, Peoples SL, Petras D, Preuss ML, Pruesse E, Rasmussen LB, Rivers A, Robeson MS 2nd, Rosenthal P, Segata N, Shaffer M, Shiffer A, Sinha R, Song SJ, Spear JR, Swafford AD, Thompson LR, Torres PJ, Trinh P, Tripathi A, Turnbaugh PJ, Ul-Hasan S, van der Hooft JJJ, Vargas F, Vázquez-Baeza Y, Vogtmann E, von Hippel M, Walters W, Wan Y, Wang M, Warren J, Weber KC, Williamson CHD, Willis AD, Xu ZZ, Zaneveld JR, Zhang Y, Zhu Q, Knight R, Caporaso JG (2019) Reproducible, interactive, scalable and extensible microbiome data science using QIIME 2. Nat Biotechnol 37:852-857

Bybee-Finley KA, Ryan MR (2018) Advancing intercropping research and practices in industrialized agricultural landscapes. Collect FAO Agric 8:80

Callahan BJ, McMurdie PJ, Rosen MJ, Han AW, Johnson AJA, Holmes SP (2016) DADA2: High-resolution sample inference from Illumina amplicon data. Nat Methods 13:581-583

Cleland EE (2011) Biodiversity and ecosystem stability. Nature education knowledge 3:14

Contreras-Cornejo HA, Macías-Rodríguez L, del-Val E, Larsen J (2018) The root endophytic fungus Trichoderma atroviride induces foliar herbivory resistance in maize plants. Appl Soil Ecol 124:45-53

Cook SM, Khan ZR, Pickett JA (2007) The use of push-pull strategies in integrated pest management. Annu Rev Entomol 52:375-400 
Dang K, Gong X, Zhao G, Wang H, Ivanistau A, Feng B (2020) Intercropping alters the soil microbial diversity and community to facilitate Nitrogen assimilation: a potential mechanism for increasing proso millet grain yield. Front. Microbiol 11:2975

Debenport SJ, Assigbetse K, Bayala R, Chapuis-Lardy L, Dick RP, McSpadden Gardener BB (2015) Association of shifting populations in the root zone microbiome of millet with enhanced crop productivity in the Sahel region (Africa). Appl Environ Microbiol 81:2841-2851

Díaz-González S, Marín P, Sánchez R, Arribas C, Kruse J, González-Melendi P, Brunner F, Sacristán S (2020) Mutualistic fungal endophyte Colletotrichum tofieldiae Ct0861 colonizes and increases growth and yield of maize and tomato plants. Agronomy 10:1493

Duchene O, Vian J-F, Celette F (2017) Intercropping with legume for agroecological cropping systems: Complementarity and facilitation processes and the importance of soil microorganisms. A review. Agric Ecosyst Environ 240:148-161

Gao L, Liu X-M, Du Y-M, Zong H, Shen G-M (2019) Effects of tobacco-peanut relay intercropping on soil bacteria community structure. Ann Microbiol 69:1531-1536

Guangchuang Y, David S, Huachen Z, Yi G, Ggtree TT-YL (2017) ggtree: an R package for visualization and annotation of phylogenetic trees with their covariates and other associated data. Methods Ecol Evol $8: 28-36$

Hiruma K, Gerlach N, Sacristán S, Nakano RT, Hacquard S, Kracher B, Neumann U, Ramírez D, Bucher M, O'Connell RJ, Schulze-Lefert P (2016) root endophyte Colletotrichum tofieldiae confers plant fitness benefits that are phosphate status dependent. Cell 165:464-474

Iqbal M, Dubey M, McEwan K, Menzel U, Franko MA, Viketoft M, Jensen DF, Karlsson M (2018) Evaluation of Clonostachys rosea for control of plant-parasitic nematodes in soil and in roots of carrot and wheat. Phytopathology 108:52-59

Khan AL, Hamayun M, Waqas M, Kang S-M, Kim Y-H, Kim D-H, Lee I-J (2012) Exophiala sp.LHL08 association gives heat stress tolerance by avoiding oxidative damage to cucumber plants. Biol Fertil Soils 48:519-529

Khan Z, Hassanali A, Pickett J, Wadhams L, Muyekho F (2003) Strategies for control of cereal stemborers and striga weed in maize-based farming systems in eastern Africa involving "push-pull" and alleopathic tactics, respectively. Afr Crop Sci Conf Proc 6:602-608

Khan Z, Midega CAO, Hooper A, Pickett J (2016) Push-Pull: Chemical ecology-based integrated pest management technology. J Chem Ecol 42:689-697

Khan ZR, Hassanali A, Overholt W, Khamis TM, Hooper AM, Pickett JA, Wadhams LJ, Woodcock CM (2002) Control of witchweed Striga hermonthica by intercropping with Desmodium spp., and the 
mechanism defined as allelopathic. J Chem Ecol 28:1871-1885

Khan ZR, Midega CAO, Bruce TJA, Hooper AM, Pickett JA (2010) Exploiting phytochemicals for developing a "push-pull" crop protection strategy for cereal farmers in Africa. J Exp Bot 61:4185-4196

Khan ZR, Midega CAO, Hassanali A, Pickett JA, Wadhams LJ, Wanjoya A (2006) Management of witchweed, Striga hermonthica, and stem borers in sorghum, Sorghum bicolor, through intercropping with greenleaf desmodium, Desmodium intortum. Int J Pest Manage 52:297-302

Khan ZR, Midega CAO, Hutter NJ, Wilkins RM, Wadhams LJ (2006) Assessment of the potential of Napier grass (Pennisetum purpureum) varieties as trap plants for management of Chilo partellus. Entomol Exp Appl 119:15-22

Koch H, Lücker S, Albertsen M, Kitzinger K, Herbold C, Spieck E, Nielsen PH, Wagner M, Daims H (2015) Expanded metabolic versatility of ubiquitous nitrite-oxidizing bacteria from the genus Nitrospira. Proc Natl Acad Sci U S A 112:11371-11376

Kuhn M, Wickham H (2020) recipes: Preprocessing tools to create design matrices $\mathrm{R}$ package version 1.8.0 https://github.com/tidymodels/recipes. Accessed 01 Oct 2020

Li Q, Chen J, Wu L, Luo X, Li N, Arafat Y, Lin S, Lin W (2018) Belowground Interactions impact the soil bacterial community, soil fertility, and crop yield in maize/peanut intercropping systems. Int J Mol Sci 19

Li T, Liu MJ, Zhang XT, Zhang HB, Sha T, Zhao ZW (2011) Improved tolerance of maize (Zea mays L.) to heavy metals by colonization of a dark septate endophyte (DSE) Exophiala pisciphila. Sci Total Environ 409:1069-1074

Love MI, Huber W, Anders S (2014) Moderated estimation of fold change and dispersion for RNA-seq data with DESeq2. Genome Biol 15:550

Macías-Rubalcava ML, Hernández-Bautista BE, Jiménez-Estrada M, González MC, Glenn AE, Hanlin RT, Hernández-Ortega S, Saucedo-García A, Muria-González JM, Anaya AL (2008) Naphthoquinone spiroketal with allelochemical activity from the newly discovered endophytic fungus Edenia gomezpompae. Phytochem 69:1185-1196

Maxwell JO, Charles AOM, Meshack O, Zeyaur RK (2018) Impact of companion cropping on incidence and severity of maize ear rots and mycotoxins in Western Kenya. Afr J Agric Res 13:2224-2231

McDonald D, Price MN, Goodrich J, Nawrocki EP, DeSantis TZ, Probst A, Andersen GL, Knight R, Hugenholtz P (2012) An improved Greengenes taxonomy with explicit ranks for ecological and evolutionary analyses of bacteria and archaea. ISME J 6:610-618

McMurdie PJ, Holmes S (2013) phyloseq: An R package for reproducible interactive analysis and graphics of microbiome census data. PLoS ONE 8:e61217 
Midega CAO, Khan ZR, Amudavi DM, Pittchar J, Pickett JA (2010) Integrated management of Striga hermonthica and cereal stemborers in finger millet (Eleusine coracana (L.) Gaertn.) through intercropping with Desmodium intortum. Int J Pest Manage 56:145-151

Midega CAO, Pittchar JO, Pickett JA, Hailu GW, Khan ZR (2018) A climate-adapted push-pull system effectively controls fall armyworm, Spodoptera frugiperda (J E Smith), in maize in East Africa. Crop Prot 105:10-15

Miller JR, Cowles RS (1990) Stimulo-deterrent diversion: A concept and its possible application to onion maggot control. J Chem Ecol 16:3197-3212

Munasinghe MVK, Kumar NS, Jayasinghe L, Fujimoto Y (2017) Indole-3-acetic acid production by Colletotrichum siamense, an endophytic fungus from Piper nigrum leaves. J Biol Active Prod Nat 7:475479

Mutyambai DM, Bass E, Luttermoser T, Poveda K, Midega CAO, Khan ZR, Kessler A (2019) More than "push" and "pull"? plant-soil feedbacks of maize companion cropping increase chemical plant defenses against herbivores. Front Ecol \& Evol 7:217

Nikandrow A (1990) Acrocalymma medicaginis and Phomopsis sp. as causal agents of crown rot of lucerne in Australia. J Phytopathol 130:24-36

Nilsson RH, Larsson K-H, Taylor AFS, Bengtsson-Palme J, Jeppesen TS, Schigel D, Kennedy P, Picard K, Glöckner FO, Tedersoo L, Saar I, Kõljalg U, Abarenkov K (2019) The UNITE database for molecular identification of fungi: handling dark taxa and parallel taxonomic classifications. Nucleic Acids Res 47:D259-D264

Njeru NK, Midega CAO, Muthomi JW, Wagacha JM, Khan ZR (2020) Impact of push-pull cropping system on pest management and occurrence of ear rots and mycotoxin contamination of maize in western Kenya. Plant Pathol 69:1644-1654

Nourbakhsh F, Koocheki A, Mahallati MN (2019) Investigation of biodiversity and some of the ecosystem services in the intercropping of corn, soybean and marshmallow. Int $\mathrm{J}$ of Plant Prod 13:35-46

Oksanen J, Blanchet FG, Friendly M, Kindt R, Legendre P, McGlinn D, Minchin PR, O'Hara RB, Simpson GL, Solymos P, Stevens MHH, Szoecs E, Wagner H (2020) vegan: Community ecology package. R package https://github.com/vegandevs/vegan. Accessed 01 Oct 2020

Palumbo JD, O'Keeffe TL, Abbas HK (2008) Microbial interactions with mycotoxigenic fungi and mycotoxins. Toxin Rev 27:261-285

Paulson JN, Stine OC, Bravo HC, Pop M (2013) Differential abundance analysis for microbial marker-gene surveys. Nat Methods 10:1200-1202 
Pedersen TL (2020) ggforce: Accelerating "ggplot2". R package https://github.com/thomasp85/ggforce. Accessed 01 Oct 2020

Pedregosa F, Varoquaux G, Gramfort A, Michel V, Thirion B, Grisel O, Blondel M, Prettenhofer P, Weiss R, Dubourg V, et al (2011) Scikit-learn: machine learning in Python. J of Machine Learning Res 12:28252830

Phillips A (2002) Botryosphaeria species associated with diseases of grapevine in Portugal. Phytopathol Mediterr 41:3-18

Poveda J, Zabalgogeazcoa I, Soengas P, Rodríguez VM, Cartea ME, Abilleira R, Velasco P (2020) Brassica oleracea var. acephala (kale) improvement by biological activity of root endophytic fungi. Sci Rep 10:20224

Prieto I, Violle C, Barre P, Durand J-L, Ghesquiere M, Litrico I (2015) Complementary effects of species and genetic diversity on productivity and stability of sown grasslands. Nat Plants 1:15033

Quast C, Pruesse E, Yilmaz P, Gerken J, Schweer T, Yarza P, Peplies J, Glöckner FO (2013) The SILVA ribosomal RNA gene database project: improved data processing and web-based tools. Nucleic Acids Res 41:D590-6

Ravnskov S, Jensen B, Knudsen IMB, Bødker L, Funck Jensen D, Karliński L, Larsen J (2006) Soil inoculation with the biocontrol agent Clonostachys rosea and the mycorrhizal fungus Glomus intraradices results in mutual inhibition, plant growth promotion and alteration of soil microbial communities. Soil Biol Biochem 38:3453-3462

R Core Team (2020) R: A language and environment for statistical computing. R Foundation for Statistical Computing, Vienna, Austria. Available online at https://www.R-project.org/

Reva ON, Swanevelder DZH, Mwita LA, Mwakilili AD, Muzondiwa D, Joubert M, Chan WY, Lutz S, Ahrens $\mathrm{CH}$, Avdeeva LV, Kharkhota MA, Tibuhwa D, Lyantagaye S, Vater J, Borriss R, Meijer J (2019) Genetic, epigenetic and phenotypic diversity of four Bacillus velezensis strains used for plant protection or as probiotics. Front Microbiol 10:2610

Ritchie ME, Phipson B, Wu D, Hu Y, Law CW, Shi W, Smyth GK (2015) Limma powers differential expression analyses for RNA-sequencing and microarray studies. Nucl Acids Res 43:e47

Rosenfeld JS (2002) Functional redundancy in ecology and conservation. Oikos 98:156-162

Sarrocco S, Mauro A, Battilani P (2019) Use of competitive filamentous fungi as an alternative approach for mycotoxin risk reduction in staple cereals: state of art and future perspectives. Toxins 11

Schnitzer SA, Klironomos JN, Hillerislambers J, Kinkel LL, Reich PB, Xiao K, Rillig MC, Sikes BA, Callaway RM, Mangan SA, van Nes EH, Scheffer M (2011) Soil microbes drive the classic plant diversity- 
productivity pattern. Ecol 92:296-303

Slippers B, Fourie G, Crous PW, Coutinho TA, Wingfield BD, Carnegie AJ, Wingfield MJ (2004) Speciation and distribution of Botryosphaeria spp. on native and introduced Eucalyptus trees in Australia and South Africa. Studies in Mycology 50:343-358

Sreevidya M, Gopalakrishnan S, Melø TM, Simic N, Bruheim P, Sharma M, Srinivas V, Alekhya G (2015) Biological control of Botrytis cinerea and plant growth promotion potential by Penicillium citrinum in chickpea (Cicer arietinum L.). Biocontrol Sci Technol 25:739-755

Stagnari F, Maggio A, Galieni A, Pisante M (2017) Multiple benefits of legumes for agriculture sustainability: an overview. Chem \& Biol Tech in Agric 4:2

Stephens M, Carbonetto P, Dai C, Gerard D, Lu M, Sun L, Willwerscheid J, Xiao N, Zeng M (2020) ashr: Methods for adaptive shrinkage, using empirical Bayes. R package https://github.com/stephens999/ashr. Accessed 01 Oct 2020

Tang X, Zhong R, Jiang J, He L, Huang Z, Shi G, Wu H, Liu J, Xiong F, Han Z, Tang R, He L (2020) Cassava/peanut intercropping improves soil quality via rhizospheric microbes increased available nitrogen contents. BMC Biotechnol 20:13

van der Heijden MGA, Bardgett RD, van Straalen NM (2008) The unseen majority: soil microbes as drivers of plant diversity and productivity in terrestrial ecosystems. Ecol Lett 11:296-310

Venkatesh N, Keller NP (2019) Mycotoxins in conversation with bacteria and fungi. Front Microbiol 10:403

Wang G, Bei S, Li J, Bao X, Zhang J, Schultz PA, Li H, Li L, Zhang F, Bever JD, Zhang J (2020) Soil microbial legacy drives crop diversity advantage: Linking ecological plant-soil feedback with agricultural intercropping. J Appl Ecol 186:281

Wickham H, Averick M, Bryan J, Chang W, McGowan LD, François R, Grolemund G, Hayes A, Henry L, Hester J, Kuhn M, Pedersen TL, Miller E, Bache SM, Müller K, Ooms J, Robinson D, Seidel DP, Spinu V, Takahashi K, Vaughan D, Wilke C, Woo K, Yutani H (2019) Welcome to the tidyverse. J Open Source Software 4:1686

Wickham H (2016) ggplot2: Elegant Graphics for Data Analysis. Springer-Verlag, New York

Wingett SW, Andrews S (2018) FastQ Screen: A tool for multi-genome mapping and quality control. F1000Res 7:1338

Xu Q, Hatt S, Lopes T, Zhang Y, Bodson B, Chen J, Francis F (2018) A push-pull strategy to control aphids combines intercropping with semiochemical releases. J Pest Sci 91:93-103 
Zin NA, Badaluddin NA (2020) Biological functions of I. for agriculture applications. Sci Ann Univ Agric Sci Vet Med 65:168-178

\section{Figures}

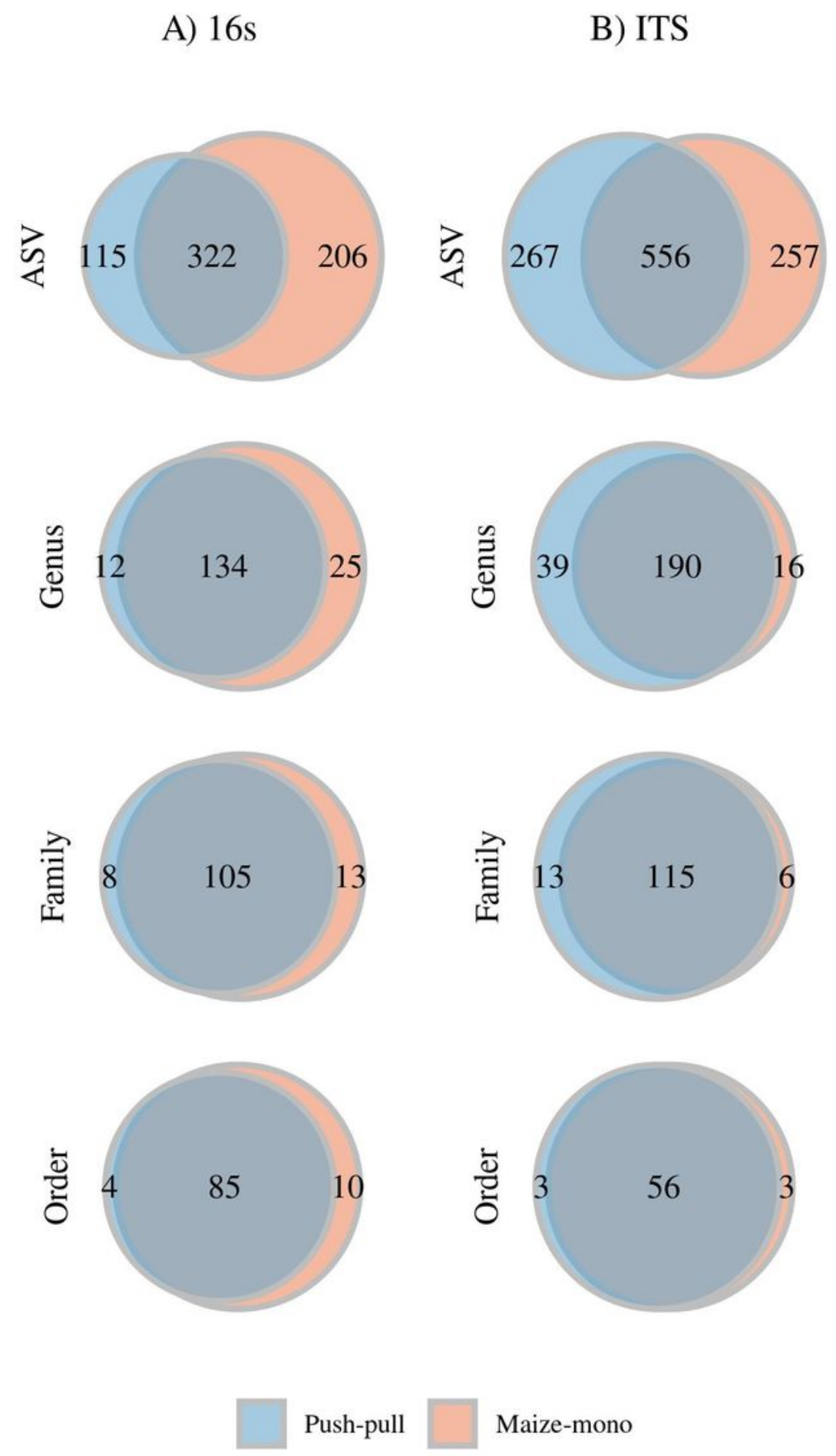

Figure 1 
Venn diagrams showing unique and common taxonomic units (ASVs, genera, families and order) for A) bacteria and B) fungi in maize-desmodium intercropping (push-pull) and maize monoculture plots. Only groups that were found more than once in the dataset were included
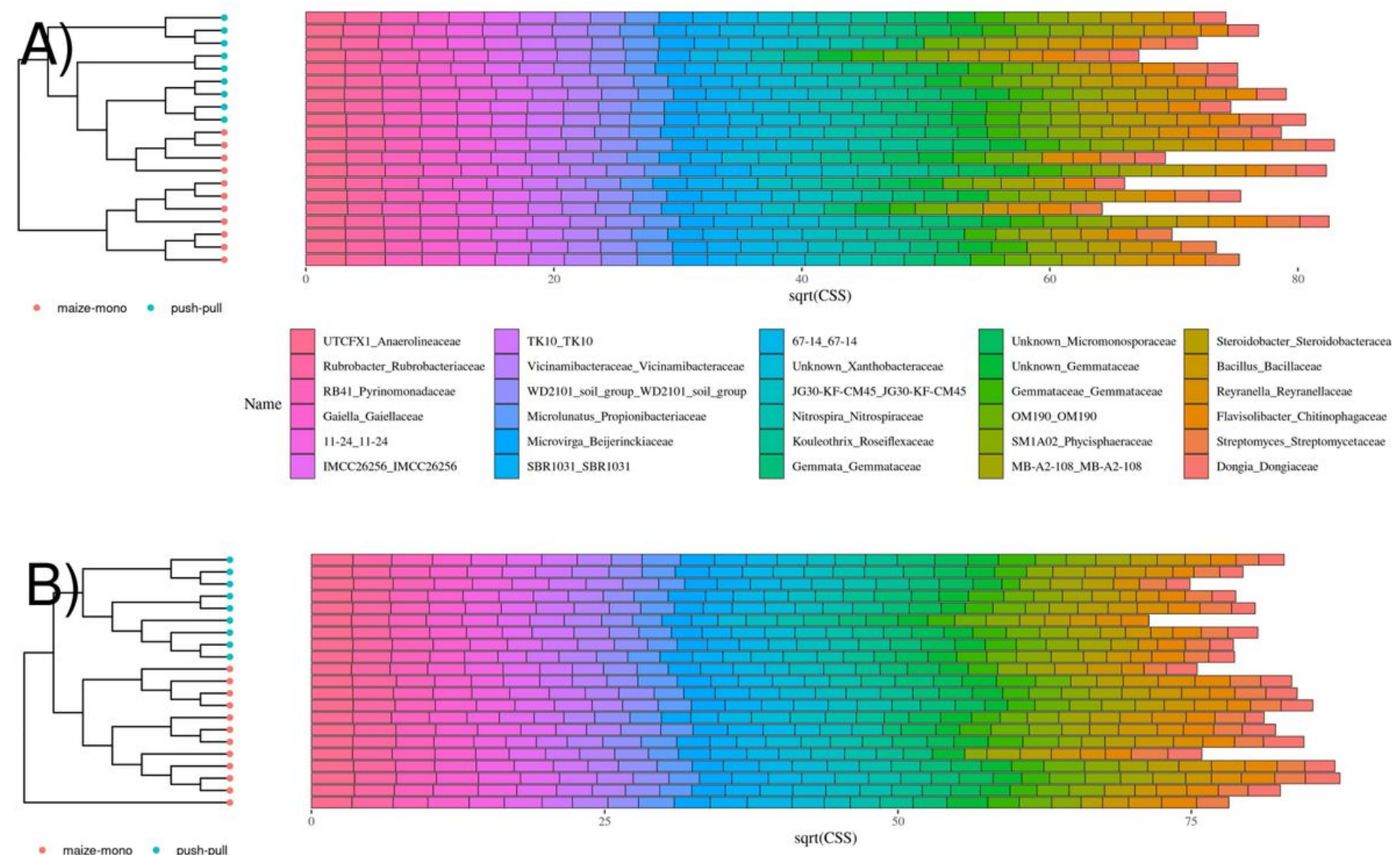

- maize-mono - push-pull

\begin{tabular}{|c|c|c|c|c|c|}
\hline \multirow{6}{*}{ Name } & Fusarium & Talaromyces & Cortinarius & Unknown_Lasiosphaeriaceae & Schizothecium \\
\hline & Didymella & Edenia & Periconia & Poaceascoma & Glomus \\
\hline & Chaetomium & Curvularia & Stachybotrys & Hannaella & Parastagonospora \\
\hline & Unknown_Chaetomiaceae & Cladosporium & Acericola & Unknown_Didymellaceac & Phacosphaeria \\
\hline & Cladorrhinum & Mortierella & Albifimbria & Preussia & Unknown_Phaeosphaeriaceae \\
\hline & Aspergillus & Coniocessia & Papiliotrema & Staphylotrichum & Achroiostachys \\
\hline
\end{tabular}

Figure 2

Bar plots showing relative abundance of soil bacterial (A) and fungal (B) genera in maize-desmodium intercropping (push-pull) and maize monoculture plots. Presented on the left are distances between samples calculated using a Jaccard dissimilarity index and presented as a dendrogram 


\section{A) $16 \mathrm{~s}$}
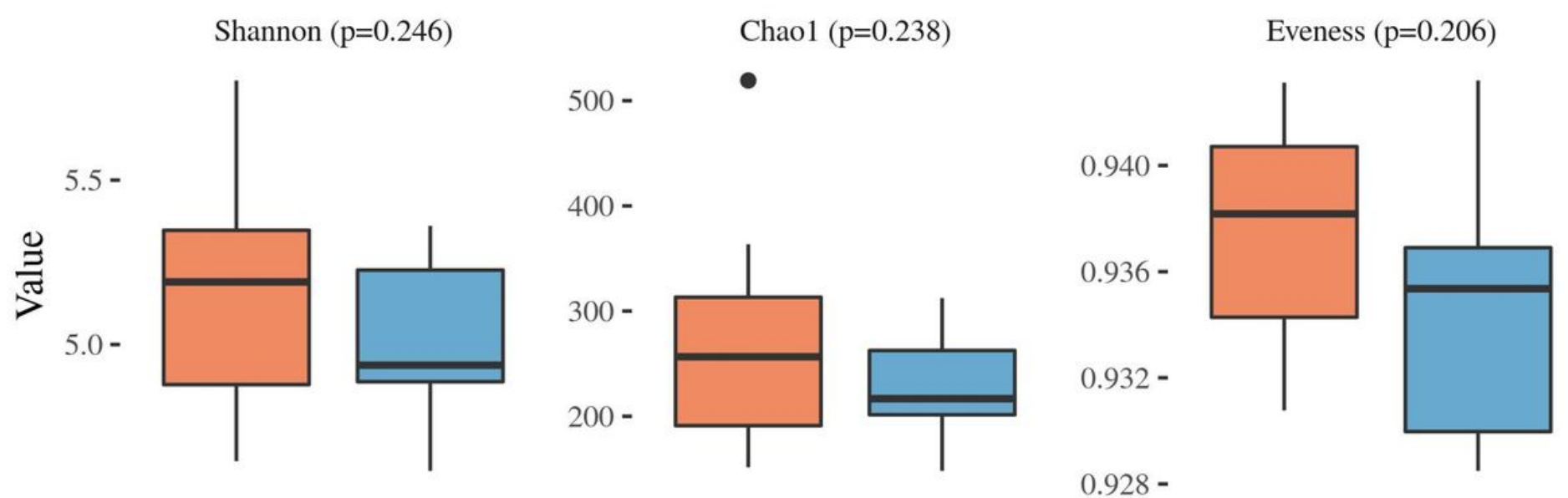

B) ITS

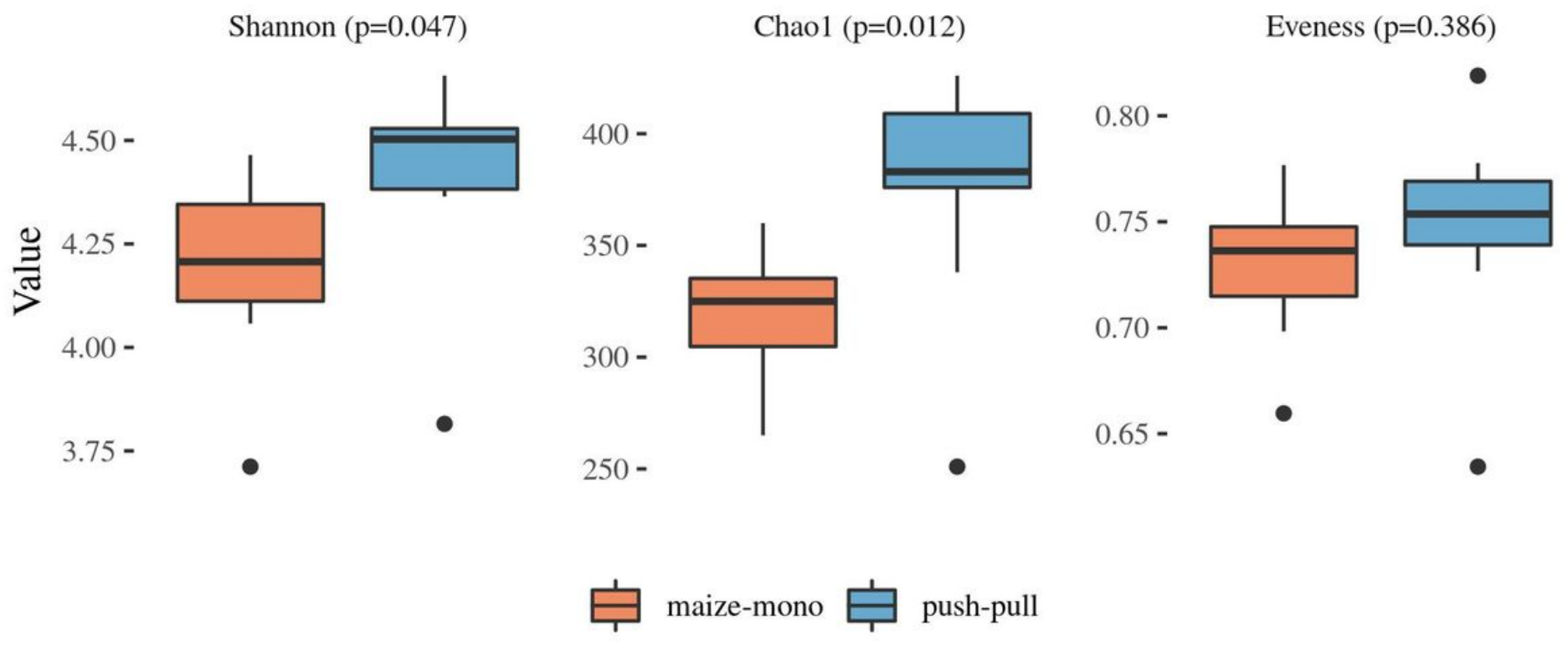

\section{Figure 3}

The diversity index (shannon), richness (chao1) and evenness (shannon/In[number of species]) of bacterial $(A)$ and fungal (B) genera across monoculture and maize-desmodium intercropping (push-pull) plots and p-values (Student's t-test) 
A) $16 \mathrm{~s}$

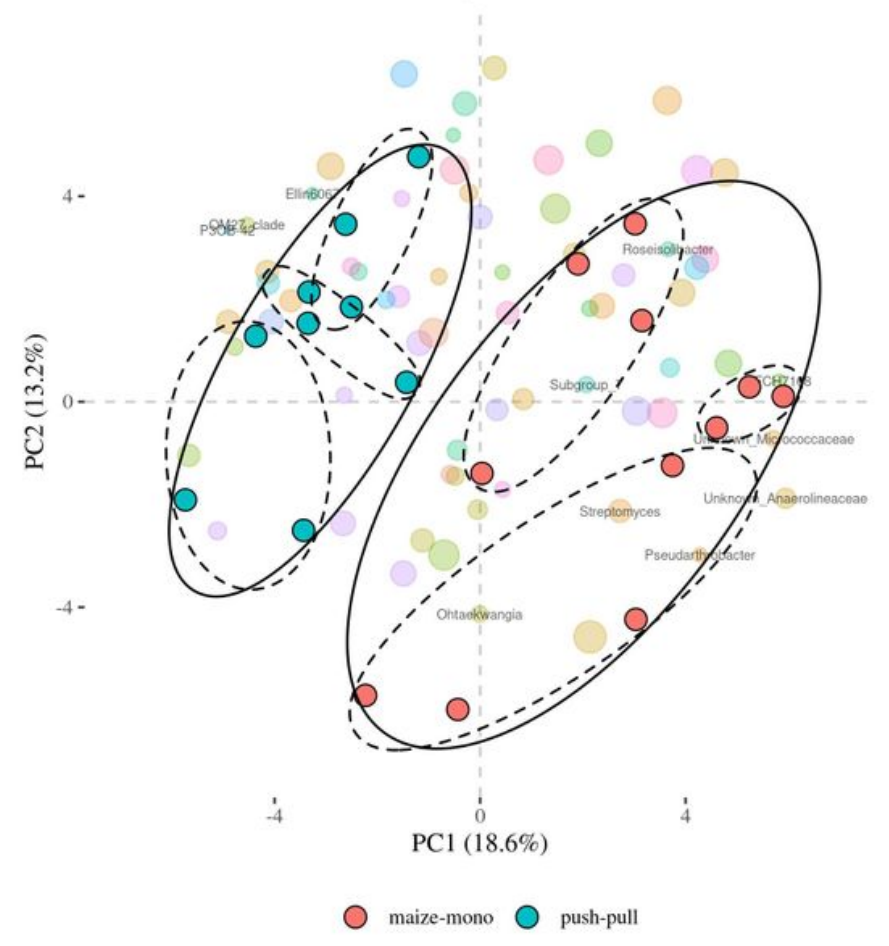

B) ITS

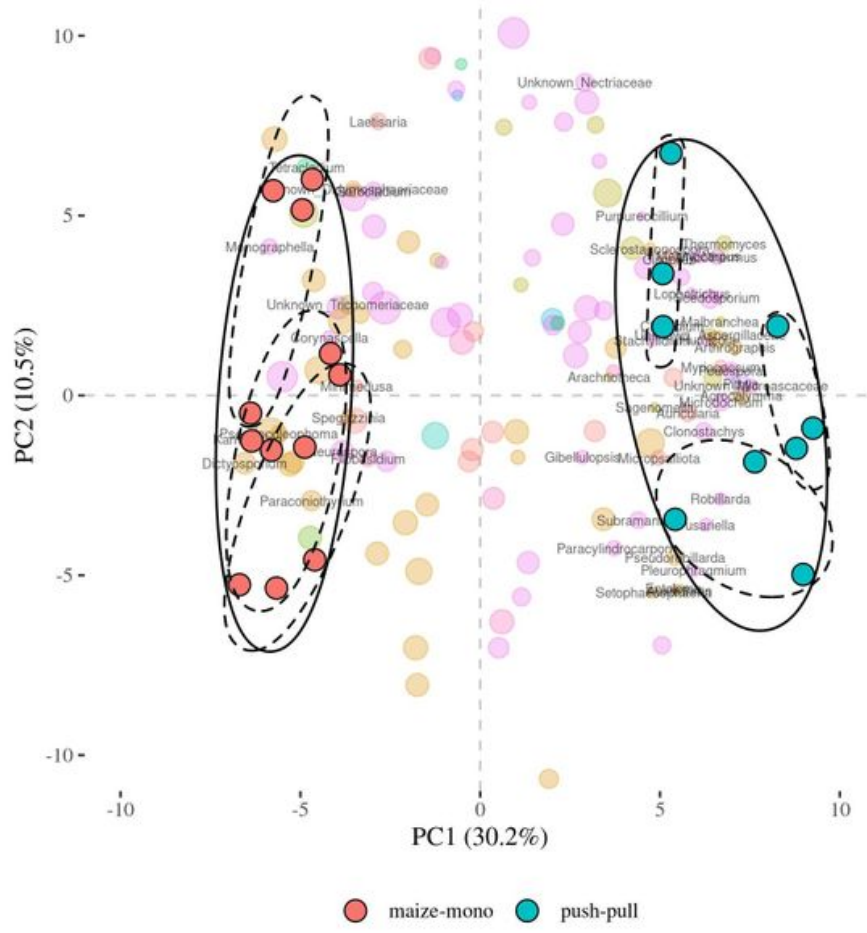

\section{Figure 4}

Principal coordinate analysis of bacterial (A) and fungal genera (B) across maize monoculture compared to maize-desmodium intercropping (push-pull). Solid ellipses around each treatment were drawn using the Khachiyan algorithm. Dotted ellipses represent samples from the same area. In the background are the eigenvectors scaled up and colored according to family 

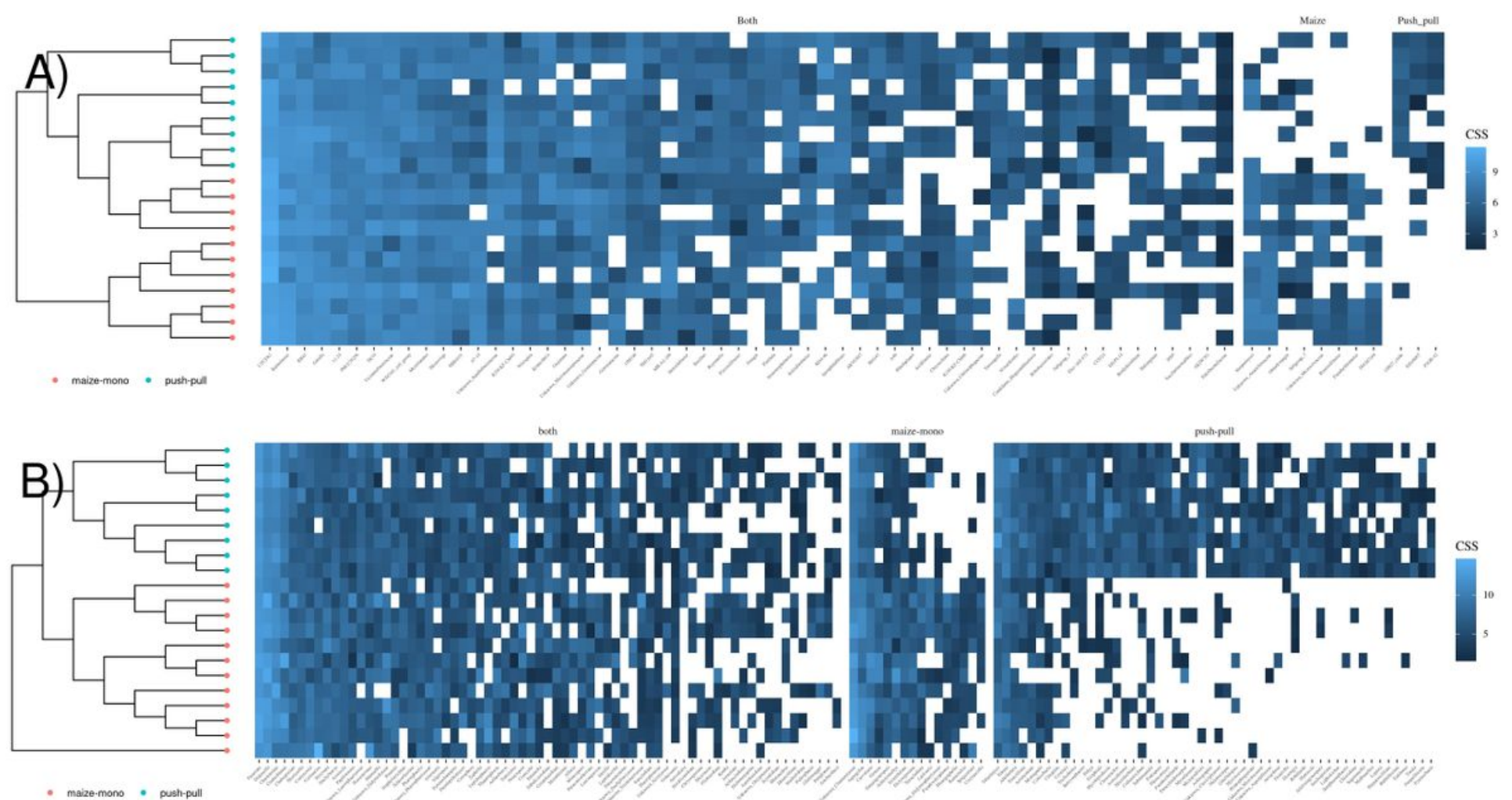

\section{Figure 5}

Heatmap showing normalized CSS (cumulative sum scaling) abundance across genera of bacteria 16s (top) and fungal ITS (bottom) in monoculture and maize-desmodium intercropping (push-pull) plots. Each horizontal line represents a different plot. The data are horizontally grouped by 'shared', 'maize' (monoculture) and 'push-pull' and within these from left-to-right by decreasing summed abundance. The dendrogram on the left is produced using a Jaccard dissimilarity index, with a presence-absence standardization. Colors indicate treatments with red representing monoculture and blue push-pull (maizedesmodium intercropping) 


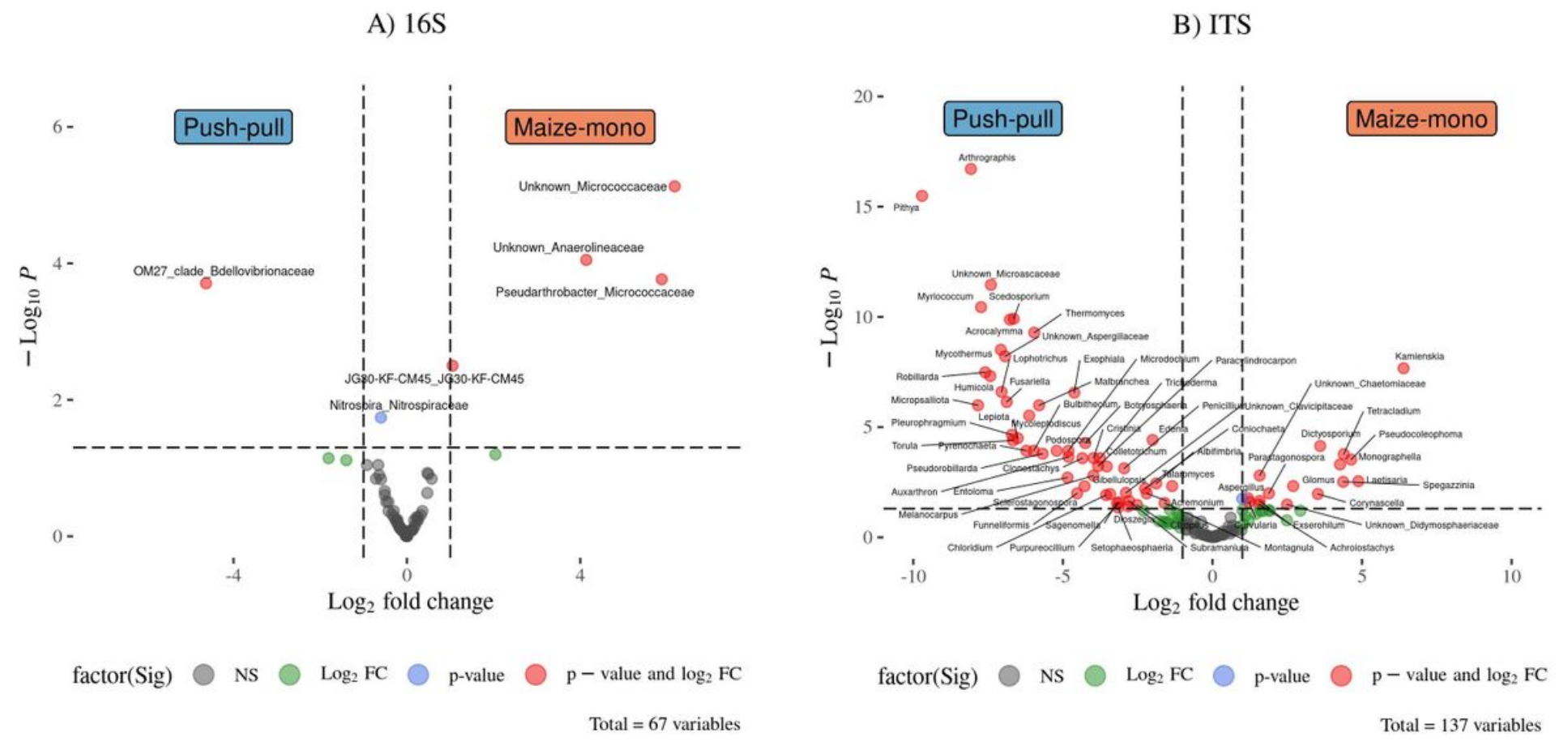

Figure 6

Volcano plots showing bacterial (left) and fungal (right) genus level features that are differentially abundant in maize-desmodium intercropping (push-pull) and monoculture plots. Genus fold changes are shown on X-axis and the negative logarithm (base 10) of the p-value resulting from a Wald test on a generalized linear model using a differential negative binomial analysis on the $\mathrm{Y}$-axis. Red dots represent genus entities that are significantly abundant in each group and with an absolute log2 fold change over 1 , with genera on the negative side of X-axis being significantly abundant in maize-desmodium intercropping (push-pull) plots. The grey and green dots represent the genus features whose abundance is similar between the two farming systems and the blue dots represents values where the $p$-value is significant between the treatments but where the log2 fold change is smaller than one

\section{Supplementary Files}

This is a list of supplementary files associated with this preprint. Click to download.

- Supplementarymaterials.docx 